\title{
Interaction communicative sur le forum de discussion en ligne français: une analyse des principes de coopération et de politesse
}

\author{
${ }^{1}$ Widya PUSPITA, ${ }^{2}$ Dudung GUMILAR \\ 1,2 Département de Français, Universitas Pendidikan Indonesia - Indonésie \\ Reçu le 3 mai 2019 | Acceptéle 30 juin 2019
}

\begin{abstract}
RÉSUMÉ. Cette étude tente d'examiner le principe de coopération et de politesse des interactions communicatives en ligne. L'étude est réalisée en utilisant la méthode descriptive qualitative. L'objet de cette recherche est un forum de discussion en ligne français. Les données sont obtenues à partir de toutes les conversations dans dix sujets sous le thème "Culture". Les résultats montrent que la discussion de tous les sujets contient le principe de coopération. Alors que le principe de politesse ne se retrouve que sur sept sujets. En outre, les résultats montrent également que le plus grand nombre de violations du principe de coopération sont les maximes de quantité et de qualité. Considérant que les violations du principe de politesse se trouvent dans la maxime de générosité. Cette recherche devrait fournir des informations complètes sur le phénomène du principe de coopération et de politesse dans les interactions communicatives indirectes, dans ce cas sur le forum de discussion en ligne.
\end{abstract}

Mots-clés: conversation en ligne, interaction en ligne, principe de coopération, principe de politesse, pragmatique

\begin{abstract}
This study attempts to investigate the cooperative and politeness principle of online communicative interactions. The study is conducted by using the descriptive qualitative method. The object of this research is an online French discussion forum. The data is obtained from all conversations in ten topics under the theme "Culture". The findings show that discussion of all topics contains the cooperative principle. While the politeness principle is only found on seven topics. In addition, the findings also show that the most violations that occurred on the cooperative principle are the maxims of quantity and quality. Whereas violations of the politeness principle are found in the maxim of generosity. This research is expected to provide comprehensive information on the phenomenon of the cooperative and politeness principle in an indirect communication interactions, in this case on the online discussion forum.
\end{abstract}

Keywords: cooperative principle, online conversation, online interaction, politeness principle, pragmatics

娄 auteur correspondant : wpuspita46@gmail.com

Pour citer cet article (Style APA) : Puspita \& Gumilar. (2019). Interaction communicative sur le forum de discussion en ligne français: une analyse des principes de coopération et de politesse. Francisola: Revue Indonésienne de la langue et la littérature françaises, 4(1), 95-105. doi: 10.17509/francisola.v4i1.20345. 


\section{INTRODUCTION}

Les violations $\mathrm{du}$ principe de coopération et de politesse se produisent dans une interaction de communication. Ces violations, par exemple, se produisent lors du choix de la forme de parole (Yusri, 2015). Cela se produit parce que quelqu'un utilise un discours difficile à comprendre, long et obscur, voire irrationnel. Comme dans l'opinion ci-dessus, nous pouvons affirmer que la violation survient lorsque le locuteur et l'interlocuteur ne se comprennent pas en totalité (Hermaliza, 2014; Setiawan 2014). Par conséquent, dans la communication, les règles stipulent que les intervenants sont nécessaires pour pouvoir travailler ensemble afin de réaliser un bon processus, de sorte que la communication puisse finalement être réalisée (Tarigan, cité dans Setiawan, 2014, p.38).

La communication peut se faire directement ou en face à face, ou indirectement par les médias sociaux par exemple. Les médias sociaux facilitent les interactions et renforcent les liens d'amitié, comme nous pouvons le voir sur Facebook, Twitter, Instagram, etc.

Des recherches portant sur les violations du principe de coopération et de politesse dans les médias sociaux ont été menées par Yulaehah (2012), qui a examiné le principe de coopération en matière de communication sur Facebook. Sur la base de la recherche, nous pouvons constater que la violation du principe de coopération mise en œuvre par l'interlocuteur indique que la communication ne fonctionnait pas correctement et qu'il $y$ avait une communication non ciblée (communication manquée). La même recherche sur les violations du principe de coopération et de politesse sur Facebook a été menée par Tussolekha, Karomani, Rusminto (2014). Les résultats de cette étude indiquent que la violation du principe de coopération et de politesse que l'on retrouve le plus souvent dans le statut Facebook est la maxime de la pertinence et la maxime de l'accord. Cela est dû à l'existence de certains objectifs, insinuant, insultant, exprimant le ressentiment, à l'existence d'une compréhension mutuelle et à l'existence de facteurs de proximité entre les interlocuteurs et les interlocuteurs.

Les non-conformités et les violations du principe de coopération et de principe de politesse sont toujours un phénomène qui se produit. Par conséquent, l'étude de cette recherche est toujours pertinente. Cette étude porte sur l'analyse du principe de coopération et $\mathrm{du}$ principe de politesse en termes d'aspects de conformité, de nonconformité et de violations à partir d'un forum de discussion en ligne. Les études mentionnées ci-dessus n'ont pas abordé l'aspect de la communication d'une plateforme de forum de discussion où, bien entendu, les modèles de communication et les messages de communication présentés sur une plateforme présentent des similitudes et des différences. Par conséquent, une étude de l'analyse des principes coopératifs et du principe de politesse sur les forums de discussion en ligne sur les médias sociaux est importante et pertinente.

Parlant du principe de coopération, selon Grice (1975), il a constaté que le principe de coopération repose sur quatre maximes : maxime de quantité, maxime de qualité, maxime de relation et maxime de manière. Dans la maxime de quantité, le participant au discours doit apporter une contribution adéquate, tandis que la maxime de qualité exige de l'interlocuteur qu'il fournisse des informations correctes et conformes aux faits. La maxime de relation stipule que, dans cette maxime, le participant $\mathrm{du}$ discours doit contribuer de manière pertinente au problème traité, tandis que la maxime de manière oblige le participant à parler directement, clairement et sans ambiguïté.

En outre, selon Leech (1983), le principe de politesse est lié aux relations entre les participants à la communication, à savoir les locuteurs et les interlocuteurs. Leech a divisé le principe de politesse en six maximes comprenant: maxime de tact, maxime de générosite, maxime 
membres du forum n'obéissent

d'approbation, maxime de modestie, maxime $\mathrm{d}$ 'accord et maxime de sympathie. Une brève explication de ces six maximes est comme suit: 1) la maxime de tact qui exige de participant qu'il maximise les profits des autres; 2) la maxime de générosite, on s'attend à ce que le discours ne soit pas moqueur; 3) la maxime d'approbation, considérée comme polie par les autres parties; 4) la maxime de modestie, le participant est censé être humble en réduisant les louanges pour lui-même; 5) maxime d'accord, lorsque chaque locuteur et interlocuteur maximise la compatibilité entre eux et minimise les disparités entre eux.; 6) maxime de sympathie qui exigent que chaque participant maximise la sympathie et minimise le sentiment d'antipathie envers l'adversaire.

De bonnes interactions de communication peuvent se produire si les participants obéissent aux règles énoncées dans le principe de coopération et le principe de politesse. La communication peut être faite directement et indirectement, un exemple de communication réalisée indirectement peut être fait sur Internet. Avec Internet, les utilisateurs peuvent facilement rechercher des informations sur les réseaux sociaux. L'un d'eux est un forum en ligne. Les forums en ligne sont un outil de communauté sur Internet qui consiste généralement à recruter des utilisateurs pour discuter de sujets appréciés de chaque utilisateur dans la section de discussion du forum.

Des recherches sur les violations des principes de coopération et de politesse sur les médias sociaux doivent encore être menées sur plusieurs autres services de médias sociaux pour prouver que ces violations peuvent également se produire dans les communications indirectes. Par conséquent, les auteurs sont intéressés par l'analyse des interactions de communication sur le forum de discussion en ligne français en termes de violations du principe de coopération et de politesse. L'objectif de cette étude est afin de savoir dans quelle mesure les violations qui se produisent si les pas à ces deux principes.

\section{MÉTHODE}

Dans le cadre d'une étude, il faut une méthode conçue comme des étapes à suivre pour résoudre des problèmes et atteindre les objectifs de la recherche. La méthode utilisée dans cette étude est une analyse descriptive qualitative, car les données utilisées seront décrites sous forme de description.

\subsection{Sujet de recherche}

Le sujet de cette étude est un ensemble de conversations contenant le principe de coopération et le principe de politesse dans un forum de discussion en ligne basé en France, à savoir ForumFr. L'objet de cette étude est la conversation trouvée sur ce forum, pourtant dans cette étude, le chercheur limite l'objet de la recherche discuté uniquement au thème "Culture" contenu dans ce forum. Le thème "Culture" comprend 10 thèmes différents : cinéma, arts et artistes, photographie, philosophie, musiques, littérature, histoire, mangas, théâtre et langue française.

\subsection{Technique de collecte de données}

Conformément aux recherches qualitatives et aux types de sources de données utilisés, les techniques de collecte de données utilisées dans cette étude sont les suivantes :

\subsubsection{La documentation}

Le chercheur a utilisé cette technique pour collecter des données afin d'observer et d'enregistrer des données devant être utilisées à partir d'une collection de conversations sur ce forum, à savoir 10 sujets contenus dans le thème «Culture».

\subsubsection{Prise de note}

Les chercheurs effectuent une lecture attentive des données qui vise à déterminer s'il existe des principes de coopération et des principes de politesse contenus dans la conversation. La prochaine chose à faire est 
d'enregistrer les données lues sur la carte de données préparée.

\subsection{Techniques d'analyse des données}

Miles et Hubermen (1994) révèlent que l'analyse de données qualitatives comporte trois étapes : la réduction, l'affichage et la vérification des conclusions. Ce qui suit expliquera les étapes utilisées dans cette étude.

\section{- Réduction des données}

Les chercheurs choisissent un discours conforme au principe de coopération et au principe de modestie, obtenus après lecture des commentaires de chaque sujet.

\section{- Affichage des données}

À ce stade, les chercheurs classent, dans une carte de données, la pertinence des données de parole sur la base des maximes contenues dans le principe de coopération et le principe de politesse.

- Mise en conclusion

Une fois triées et classées dans des cartes de données, la dernière étape est la mise en conclusion.

\section{RÉSULTATS ET DISCUSSION}

\subsection{Analyse des principes de coopération et de politesse}

Dans cette étude, les chercheurs ont trouvé des discours appropriés et non conformes au principe de coopération sur l'ensemble des thèmes abordés dans le forum. Cependant, sur ces 10 thèmes, les chercheurs ont trouvé le principe de politesse sur 7 thèmes, notamment : (1) Thème 3: Votre dernier livre d'histoire lu; (2) Thème 4: vous avez dessiné pour le manga naruto?; (3) Thème 5: Bonjour, qu'est-ce que ça veut dire? (4) Thème 6: Le meilleur chanteur selon vous; (5) Thème 7: Michael Jackson; (6) Thème 8: Des nouvelles, des nouvelles, des nouvelles ...; et (7) Thème 10: Quelles pièces avez-vous vues?

\subsubsection{Thème 1 : Films d'horreurs}

Contexte : Le compte de Martine demande à tous les membres du forum de fournir des recommandations ou des opinions sur des films d'horreur intéressants. Ci-dessous est un discours conforme à la maxime de quantité fondée sur le principe de coopération.

\section{Martine : Des films d'horreurs à conseiller? \\ Hannibal25 : Je te conseille tous les "Jason" y compris le petit dernier.}

Le discours d'Hannibal25 montre qu'il a répondu en fournissant des informations sous la forme de titres de films d'horreur qu'il a jugés intéressants, il l'a donc recommandée à Martine. Le discours de Hannibal25 est conforme au principe de la maxime de quantité, car il ne donne que la contribution du partenaire au discours, c'està-dire en répondant simplement aux questions de Martine sur ses opinions ou recommandations concernant les films d'horreur.

En plus d'un discours conforme aux règles de la maxime quantité, cette étude a également révélé des discours inappropriés, comme dans le dialogue suivant:

Martine : Des films d'horreurs à
conseiller?
Valentine ${ }^{*} \quad:$ Bonsoir... Huum,ça
fait un petit moment que je ne regarde
plus trop ce genre de films, je suis plus
dans les thrillers que dans le gore.

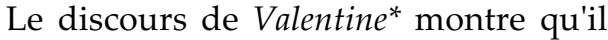
n'a pas fourni les informations dont Martine avait besoin, il ne mentionne ici que le type de film d'horreur mais n'est pas accompagné du nom du film, ce qui est un élément important de la question de Martine sur la recommandation des titres de films d'horreur. Donc, le discours de Valentine* n'est pas conforme au principe de la maxime de quantité. La phrase dans le discours montre que Valentine*, en tant que participant au discours, ne peut contribuer sous la forme d'informations dont Martine a besoin.

\subsubsection{Thème 2 : Que pensez-vous de l'intuition? \\ Contexte : le compte SandraNewLife} demande à tous les membres du forum leur opinion sur l'intuition. Ci-dessous est un 
les informations n'étaient pas

discours qui est conforme à la maxime de qualité sur le principe de coopération.

SandraNewLife : Mais devons nous suivre cette intuition, essayer même de la comprendre, de chercher à savoir le fond ?

Pila : L'intuition ne se contrôle pas, à mon avis. On devine parfois des choses mais on ne sait pas qu'elles vont arriver. La semaine dernière, j'ai pensé deux fois à des connaissances de la commune que je n'avais pas vu dans la rue depuis longtemps.

Le discours de Pila ci-dessus montre qu'il a fourni des informations sous la forme de son expérience d'intuition en racontant les événements survenus la semaine dernière à propos de la pensée d'un inconnu. Ce discours est donc conforme aux règles de la maxime de qualit, car Pila, en tant que participant au discours, fournit une information basée sur l'événement vécu directement afin que la vérité puisse être crue, car elle est basée sur des preuves ou des faits existants, qui concerne l'incident qu'il a vécu la semaine dernière. Par contre, dans les données suivantes, il existe des écarts dans la maxime de qualité.

SandraNewLife : Mais devons nous suivre cette intuition, essayer même de la comprendre, de chercher à savoir le fond ?

Mai : L'intuition peu être forte et constante concernant éventuellement une personne ou une chose qui pourrait se produire. Mais quand je parle de comprendre cette intuition c'est tout simplement essayer dans découvrir le sens. Mon intuition me dit qu' une personnes que je côtoie n'est pas celle que je crois, j'observe cette personne, écoute... et ce que je vois me trouble cela va dans le sens de mon intuition. Serait-il possible que le cerveau écoute vois et interpréte dans le sens de cette intuition ou tout simplement confirme ce ressenti ?

Le discours de Mai montre qu'il a une expérience d'intuition basée sur ses propres pensées. Sa parole n'est pas conforme aux règles de la maxime de qualité, car Mai n'a pas fourni d'informations indiquant qu'elle en avait fait l'expérience directe, de sorte que nécessairement comprises ou acceptées par SandraNewLife.

\subsubsection{Thème 3 : Votre dernier livre d'histoire lu. \\ Contexte : Le compte Nerelucia} demande à tous les membres du forum si quelqu'un qui lit des romans historiques peut donner une recommandation pour la lecture d'un autre roman.

Nerelucia : Tout le monde ici lit des romans et les amateurs d'histoire? Quel livre avez-vous lu dernièrement ? Je commence ; memoires de Gaulle, en trois tomes, je conseille.

Maxence22 : Livre d'Histoire: La guerre de Sept Ans, Edmond Dziembowski, chez Perrin.

Le discours de Maxence22 montre qu'il a donné à Nerelucia des informations sur les noms des derniers romans historiques qu'il avait lus. Son discours est donc conforme au principe de maxime de quantité, car il ne donne que la contribution du partenaire au discours (voir Grice, 1975), dans ce cas là, en fournissant des informations sur les noms des titres de romans historiques lus ou ayant été lus.

Un autre exemple de la conformité aux règles de la maxime de quantité se trouve dans l'extrait suivant:

Nerelucia : Tout le monde ici lit des romans et les amateurs d'histoire ? Quel livre avez-vous lu dernièrement? Je commence ; memoires de Gaulle, en trois tomes, je conseille.

Magus : Les derniers d'Histoire que j'ai lu sont :

-Louis XIV de Thierry

-L'année des quatre dauphins

-Louis XV de Jean

-Les bourreaux en France

Le discours de Magus montrait qu'il donnait des informations à Nerelucia sous la forme des noms des derniers livres d'histoire qu'il avait lus. Cela montre que le Magus, en tant que participant au discours, ne fournit que la contribution nécessaire en donnant des informations sur les noms des titres de livres historiques.

S'agissant le principe de politesse, nous pouvons le repérer que le discours 
suivant est celui qui est conforme au maxime de tact.

Nerelucia : Tout le monde ici lit des romans et les amateurs d'histoire? Quel livre avez-vous lu dernièrement? Je commence ; memoires de Gaulle, en trois tomes, je conseille.

Brendan12 : Le dernier que j'ai lu est "De quoi sont-ils vraiment morts ?" de Jacques Deblauwe. Un très bon bouquin donnant la vérité sur la mort de grands personnages historiques, comme Louis XIV, Mirabeau, Robespierre, Agnès Sorel, Louis IX.....C'est un peu glauque, mais c'est vraiment passionnant ! Je vous le conseille vivement !

Le discours de Brendan12 montre qu'il a lu des livres sur l'histoire des grandes figures françaises et que, selon lui, ce livre est très intéressant. Son discours est donc conforme à la maxime de tact, car dans la phrase "Je vous conseille vivement" figure la maxime de tact, car il contient un discours contraignant qui montre que l'interlocuteur maximise les bénéfices du locuteur.

\subsubsection{Thème 4 : Avez-vous besoin de dessiner pour le manga Naruto?}

Contexte : le compte Antonintendofan a des difficultés à dessiner un manga, puis il demande l'aide de tous les membres du forum si quelqu'un a une idée de sa capacité à dessiner des techniques de manga pour l'aider et le contacter immédiatement car il a un bon projet à réaliser ensemble.

Antonintendofan: Ça vous de dessiner un manga naruto??? Moi oui mais le problème je sais pas dessiner donc... Mais avec tout les autres fan de ce manga on pourait écrire un truc pas mal. Si ca vous dit dite le... On est pas obligé de savoir dessiner: certain peuvent écrire le scénario,d'autre peuvent dessiner des personnages et des décor mais aussi inventer des techniques pour les personnages qu on aura inventer. Si assez de monde veut le faire j'ai déja une tite idée scénaristique.

The_Fan: Salut, voilà j'ai réussi à te trouver deux sites: le premier est pour aprrendre à dessiner les personnages de Naruto et l'autre est un livre pour apprendre à dessiner les mangas. Bon voilà ma mission est terminée. Au fait voici les liens:

- Persos Naruto

- Livre de la FNAC

Le discours de The_Fan montre qu'il fournit ici des informations sur des sites et des livres pouvant aider Antonintendofan à dessiner des personnages de manga. Son discours est donc conforme au principe de la maxime de quantité, car The_Fan, en tant que participant, apporte tout autant que nécessaire l'interlocuteur, notamment en fournissant des informations sur des livres et des sites sur les mangas qui peuvent aider Antonintendofan à surmonter ses difficultés même s'il n'est pas ici. Antonintendofan peut participer ou recevoir une offre, mais les informations fournies par The_Fan sont conformes à ses besoins.

En outre, sur le sujet 4, certains discours sont conformes à la maxime de relation et sont visibles dans la conversation suivante :

Kakashi Hatake : Salut, c'est vrai que tout le monde aiment faire la suite des histoires selon son gout mais finalement il s'apercevront qu'ils ne font que recopier l'histoire originale, essayer plutôt de faire votre propre histoire, c'est pas facile, je vous le dit, inventé une seule personnage avec 2 techniques, c'est du travaille a faire, c'est vrai qu'il y-a des gens qui débordent d'imagination, mais ils vont avoir une limite sur leurs imaginations, dans une bd il faut avoir au moins + de 4 personnage principaux, enfin c'est le minimum, pouvez vous faire c'est 4 personnage sont qu'elle soit relier directement a Naruto ou autre manga??? C'est pourquoi, si vous le voulais, voila une histoires, créer par moi ('je ne vais pas vous aidés du début jusqu'à la fin'), je vous donne le commencement et a vous de la faire suivre, chacun a une suite.

Anastushia : Désolé mais ça reste un peu du "déjà vu" comme histoire. Ce n'est pas assez original à mon goût (attention, je ne suis pas un pro non plus) Ce n'est pas facile d'écrire un scénario, j'en ai un à moi, que je travaille depuis des années (ce n'est pas un manga et je n'y consacre pas tout mon temps non plus (d'autres projets sur le feu) mais pour que l'histoire reste alléchante 
pendant tous les albums (si c'est une histoire à suivre, bien sûr) ; il faut une longue réflexion.

Le discours d'Anashtusia montre qu'il a la même expérience que le constat de Kakashi Hatake qui parle de la difficulté de dessiner un manga. Son discours est donc conforme aux règles de maximisation pertinentes pour le principe de coopération. La phrase sur le discours montre qu'Anashtusia, en tant que participant, a fourni des informations liées au sujet traité.

S'agissant la maxime de politesse, un exemple du texte ci-dessous correspond à la maxime de tact.

Antonintendofan : Ça vous de dessiner un manga naruto? Moi oui mais le problème je sais pas dessiner donc. Mais avec tout les autres fan de ce manga on pourait écrire un truc pas mal. Si ca vous dit dite le...

On est pas obligé de savoir dessiner: certain peuvent écrire le scénario,d'autre peuvent dessiner des personnages et des décor mais aussi inventer des techniques pour les personnages qu on aura inventer. Si assez de monde veut le faire j'ai déja une tite idée scénaristique.

Mdr : Moi je sais un peu desiner des naruto je comence apeine a les desiner en faite mais si sa te dit je peux t'aider dans ton projet.

Le discours de Mdr a montré qu'elle pouvait aider Antonintendofan à dessiner des mangas, même si ce n'était pas très bon, mais au moins, pourrait aider un peu. Son discours est donc conforme à la maxime de tact, car dans la phrase "je peux t'aider dans ton projet" contient un discours commissif. Il apparaît que la phrase est centrée sur l'interlocuteur $(M d r)$, où il donne un avantage au locuteur (Antonintendofan). Le discours montre qu'il a offert son aide à Antonintendofan pour travailler ensemble sur le projet.

\subsubsection{Thème 5 : Bonjour, qu'est-ce que ça veut dire? \\ Contexte : Le compte Kokinyo demande} à tous les membres du forum ce que les mots signifient dans "création de post".
Kokinyo: Bonjour, qu'est ce que ca veut dire creation de poste? Merci beaucoup.

L'abbe resina : Sur un forum, c'est un nouveau sujet qui s'appelle post.

Le discours de L'abbe resina a montré qu'il avait répondu à la question de Kokinyo en expliquant le sens de l'expression "création de poste". Il expliquait que si dans un forum le mot équivalait au téléchargement d'un statut ou d'un nouveau sujet créé par le titulaire du compte. Le discours de L'abbe résina est conforme à la maxime de qualité, car en tant que participant au discours, il fournit des informations basées sur des événements réels, de sorte que la vérité puisse être crue, car elle est basée sur des preuves ou des faits existants (voir Grice, 1975), ce qui concerne sa déclaration sur l'explication de la phrase " "création de poste", qui est liée à la condition réelle ou réelle selon laquelle, dans l'utilisation des médias sociaux, la phrase est souvent utilisée lorsque le titulaire du compte crée un nouveau statut ou un nouveau sujet à télécharger, tels que des forums en ligne, Facebook, Twitter, etc.

Le discours suivant est celui qui est conforme à la maxime de générosité du principe de politesse.

Pinoccchio : Il parle d'une création de poste, (pas post), la venue d'un nouveau métier, je presume.

Amelane: Bonjour, merci de me corriger, c'est ça, le poste, merci beaucoup.

Le discours d'Amelane montre qu'il a remercié Pinoccchio pour avoir corrigé ses propos. Son discours est conforme à la maxime de générosité, car il maximise le respect des autres (voir Leech, 1983).

\subsubsection{Thème 6: Le meilleur chanteur selon vous.}

Contexte : le compte Cosmos demande à tous les membres du forum leur opinion sur les chanteurs qu'ils idolâtrent tant pour ceux qui sont morts que pour ceux qui sont encore en vie.

Cosmos : Salut qui est le meilleur chanteur d'apres vous q'il soit mort ou vivant. D'apres moi Michael Jackson. 
Pila : Il y en a tellement !

Alain Barrière.

Le discours de Pila a montré qu'il avait donné une réponse en mentionnant l'un de ses chanteurs d'idoles, Alain Barrière. La phrase de Pila montre que, en tant que participant parlant, contribue suffisamment à l'interlocuteur, notamment en donnant des informations sur le nom de l'un de ses chanteurs d'idoles. De plus, on peut dire que le discours est conforme à la maxime de quantité, car les informations fournies par Pila ne sont pas excessives. D'autre exemple, ci-dessous est le discours qui est conforme à la maxime d'accord.

Cosmos : Salut qui est le meilleur chanteur d'apres vous q'il soit mort ou vivant. D'apres moi Michael Jackson.

Bondgers : Patriccck !!!! Heu, sinon moi j'étais assez d'accord avec les américains quand ils avaient élu Aznavour comme meilleur chanteur du 20ème siècle. Mais bon tu as moins de 20ans alors tu ne peux pas connaitre.

Le constat de Bondgers montre qu'il a mentionné un chanteur de variété française qu'il apprécie. Son discours n'est pas donc conforme à la maxime d'accord. Cela est démontré par l'expression "mais bon tu as moins de 20ans alors tu ne peux pas connaitre" qui appartient à la catégorie de violation des règles de la maxime d'accord, car l'interlocuteur ne maximise pas la compatibilité avec ce qui est parlé par le locuteur (voir Leech, 1983). Ceci est dû au fait que dans le discours, Bondgers parle de vieux chanteurs que Cosmos ne connaît pas forcément, de sorte que le discours peut conduire à une inégalité de perception.

\subsubsection{Thème 7: Michael Jackson.}

Contexte : le compte Hannibal25 demande à tous les membres du forum si le roi de la pop (Michael Jackson) est vraiment mort ou non, car de nombreux témoignages indiquent qu'il est toujours en vie.

Hannibal25 : Pensez vous que le roi de la pop est vraiment mort? Plusieurs temoignages demontre qu'il es vraiment vivant que sa mort fu un coup mont.plusieurs videos a l'appui.Ce mec a bercer mon enfance et aujourd'hui, je l'ecoute encore. Ma chanson preferée est THRILLER en version complete et vous?

January : : MJ est mort. Stop aux fantasmes... Et oui Elvis est mort aussi, et Kennedy, et on a marché sur la lune, et un avion s'est écrasé sur le pentagone, etc. Je comprends que c'est amusant la théorie du complot, mais pour certaines choses quand même, ça confine à l'extrême naïveté pour ne citer que cela.

D'après les données ci-dessus, nous pouvons voir que le compte d'Hannibal25 a demandé à tous les membres du forum si le roi de la pop (Michael Jackson) était vraiment mort ou non, car de nombreux témoignages affirmaient qu'il était toujours en vie. Ensuite, la question de Hannibal25 a été répondue par un compte rendu de January. La réponse de January montre qu'il a déclaré que Michael Jackson était mort, y compris d'autres personnalités célèbres. Tous ces témoignages n'étaient que des théories du complot qui ne pouvaient être acceptées par tout le monde.

Le discours de janvier est donc conforme aux principes de politesse de la maxime de tact. La phrase "stop aux fantasmes" tombe dans la catégorie de la maxime de tact, car elle contient des énoncés impérieux (voir Leech, 1983), ce qui montre que January a ordonné de cesser de rêver que de grands personnages et des artistes morts pourraient ressusciter, et de ne plus parler d'un des choses qui dépassent la raison.

\subsubsection{Thème 8: Des nouvelles, des nouvelles, des nouvelles ...}

Contexte : le compte Invité indique qu'il cherche une collection de livres de roman de certains de ses plus récents écrivains préférés. Ici, il demande l'aide de tous les membres du forum pour lui donner des idées sur les titres des livres.
Invité
: Bonsoir à tous,
Je cherche des recueils de nouvelles (pas assez patient pour lire des romans...) Mes auteurs prefers Buzzati, Bradbury, Carver, Murakami, Sturgeon. Des idées?
Pila Chroniques martiennes par Bradbury! C'est chouette.
Le discours de Pila montre qu'il a donné l'un des titres du roman de Bradburry 
visage, les téléphones, l'oiseau et

à Invité, Chroniques martiennes. Son discours est conforme aux règles de la maxime de quantité, car Pila, en tant que participant parlant, contribue suffisamment ou autant que l'interlocuteur en a besoin (voir Grice, 1975), notamment en fournissant des informations sur l'un des romans de Bradburry où Invité, qui a effectivement besoin de ces informations, cherche des recommandations pour le titre de son roman préféré.

S'agissant le principe de politesse, le corpus suivant est un exemple celui qui est conforme à la maxime de tact.

Invité : Bonsoir à tous,
Je cherche des recueils de nouvelles (pas
assez patient pour lire des romans...)
Mes auteurs prefers Buzzati, Bradbury,
Carver, Murakami, Sturgeon. Des idées?
Edgar : Si t'as aimé le King et
son univers un peu Dark, toujours en
format court, je te propose les nouvelles
d'Edgar Allan Poe, et les histoires courtes
de Lovecraft.

Le discours d'Edgar montre qu'il a formulé des recommandations concernant le titre du roman dont Invité avait besoin. Son discours est donc conforme à la maxime de tact qui est démontré par la phrase "je propose les nouvelles d'Edgar, Allan Poe et les histoires avec la permission de Lovecraft", car elle contient un discours comissif (Voir Leech, 1983). En effet, cette phrase est centrée sur l'interlocuteur où il profite au locuteur, ce qui montre qu'il recommande les romans et les nouvelles de Edgar Allan Poe de Lovecraft.

\subsubsection{Thème 9 : Photographie officielle d'Emmanuel Macron.}

Contexte : le compte rendu de January commente une photo du président français Emmanuel Macron, qui a ensuite demandé un autre avis sur la photo à tous les membres du forum.

January : Je trouve trop serré, j'aurais enlevé les portes fenêtres. Et vous, comment vous trouvez? (Attention ici on parle Photo on ne parle pas de politique merci).

Gouderien : J'aime bien le paysage extérieur, les drapeaux, la tenue de son l'horloge, mais moins le cadrage, la posture, le mobilier et le bouton ouvert de sa veste.

Le discours de Gouderien montrent qu'il a répondu à la question de January en commentant la photo. Son discours est donc conforme aux règles de la maxime de qualité. Cela est démontré par sa réponse qui fournit une information fiable, car elle est basée sur des preuves ou des faits existants (voir Grice, 1975), dans ce cas, des commentaires sur les éléments physique de la photo afin que les informations fournies puissent être considérées comme vraies.

\subsubsection{Thème 10: Quelles pièces avez- vous vues?}

Contexte : le compte Pila demande à tous les membres du forum le titre du théâtre vu de son vivant.

Pila : Quelles pièces de théâtre avezvous vu dans votre vie? En scolaire, j'ai vu "Le malade imaginaire" et "Le bourgeois gentil homme". J'ai également vu plus tard "Les justes", de Camus. Une pièce sur la révolte des bonnets rouges (Bretagne -1675). Une opérette aussi; avec Guétary.

Moor : Me reviennent; "Le dîner de cons" (Veber), "Le joueur d'échecs" (adaptation d'une nouvelle de Zweig) "Oncle Vania" + "La Mouette" (Tchekov).

Le discours de Moor a montré qu'il avait répondu en répondant à plusieurs titres de théâtre vues. Son discours est donc conforme aux règles de la maxime de quantité. La réponse de Moor montre qu'il contribue autant que le nécessite l'interlocuteur en fournissant des informations sur plusieurs titres de théâtre.

S'agissant le principe de politesse, ci-dessous est un exemple de discours qui est conforme à la maxime d'accord.

Pila : Quelles pièces de théâtre avez-vous vu dans votre vie? En scolaire, j'ai vu "Le malade imaginaire" et 
Le bourgeois gentil homme". J'ai également vu plus tard "Les justes", de Camus. Une pièce sur la révolte des bonnets rouges (Bretagne -1675). Une opérette aussi; avec Guétary. Enchantant : J'aime pas le théâtre.

Le discours d'Enchantant montre qu'il n'aime pas le théâtre. La phrase "J'aime pas le théâtre" comme réponse à la question de Pila, ne fait pas partie de la maxime d'accord, car l'interlocuteur ne maximise pas la compatibilité et l'adéquation des activités de conversation (Voir Leech, 1983). Dans ce cas là, Enchantant répond en indiquant qu'il n'aime pas le théâtre, alors que Pila demande à tous les membres du forum qui aiment uniquement le théâtre.

\section{CONCLUSION}

Sur la base des résultats de l'analyse, nous pouvons conclure que la maxime la plus fréquente est la maxime de sagesse. Cela indique que les locuteurs respectent les règles de la maxime de sagesse en essayant de minimiser les pertes pour les autres parties. Alors que la maxime qui apparaît le moins est la maxime de la générosité. La divergence de discours entre les maximes de générosité est due au fait que l'interlocuteur ne cherche pas à créditer les autres partis de manière à ce qu'il puisse être considéré comme une personne impolie.

En outre, nous avons également conclu que des violations du principe de coopération avaient été commises : la règle la plus couramment retenue étant la maxime de quantité et de qualité. Cela est dû au fait que certains membres du forum ne peuvent toujours pas fournir les informations demandées par le locuteur. En outre, les informations fournies n'étaient pas conformes aux faits et ne pouvaient donc pas faire confiance aux informations des deux parties. Semblable au principe de coopération, même dans le principe de politesse, on constate une violation de la maxime d'accord. Cela se produit car le locuteur et l'interlocuteur n'optimisent pas la compatibilité entre eux.
Les inadéquations et les violations de la parole peuvent interférer les interactions de communication dans chaque conversation conduite par les participants à la parole. Dans cette étude, seules quelques divergences et violations ont été constatées. Dans certains cas, cette inadéquation des maximes interfère certainement avec le cours des interactions de communication, ce qui peut entraîner des malentendus, des paroles qui nuisent et insultent les autres, ainsi que des informations incompréhensibles. Cette différence est l'impact des violations commises par les participants au discours parce que le discours prononcé n'est pas clair et en quoi il rend le discours impoli. De plus, l'interlocuteur exprime trop ses sentiments pour ne pas offenser le locuteur.

Cette recherche a ses limites, notamment car les objets de données ne proviennent que d'un seul forum de discussion en ligne et ne sont limités qu'à 10 sujets. Si l'objet de cette recherche est élargi en examinant davantage de forums de discussion en ligne et plus de thèmes, elle devrait pouvoir fournir des informations beaucoup plus complètes sur le phénomène du principe de coopération et de politesse dans les conversations en ligne.

\section{RÉFÉRENCES}

Grice, H.P. (1975). Logic and Conversation. Dans P. Cole et J.L. Morgan (dir.), Syntax and Semantics (Vol.3, p.41-58). New York: Academic Press.

Hermaliza. (2014). Pelanggaran Prinsip

Kerjasama dalam Tuturan Ironi Talkshow Bukan Empat Mata di TRANS 7: Tinjauan Pragmatik. Jurnal Bahasa, 9(2), 114-123. Retiré à https://ejournal.unri.ac.id/index.php/J B/article/view/2451

Leech, G. (1983). Principles of pragmatics. London; Newyork : Longman.

Miles, M. B., \& Huberman, A. M. (1994).

Qualitative Data Analysis An

Expanded Sourcebook. Thousand Oaks: CA Sage Publications.

Setiawan, F. (2014). Penggunaan Prinsip Kerja Sama dalam Kegiatan Berdiskusi 
Siswa Kelas XI IPS SMAN 1 Semin Gunungkidul. (Mémoire de licence inédit). Universitas Negeri Yogyakarta

Tussolekha, R., Karomani, K., \& Rusminto, N. E. (2014). Prinsip Kerja Sama dan Sopan Santun Siswa di Jejaring Facebook dan Implikasinya. J-SIMBOL (Bahasa, Sastra, dan Pembelajarannya), 2(1).

Yulaehah, F. (2012). Analisis Prinsip Kerja Sama pada Komunikasi Facebook (Studi Kasus pada Mahasisiwa Bahasa dan Sastra Indonesia Universitas Negri Yogyakarta Angkatan 2007). Skripsi S1.

Yusri. (2015). Pelanggaran Kesopanan Berbahasa dalam Komunikasi Politik pada Pemilihan Gubernur Sulawesi Selatan 2013. Parole, 5(1), 26-39. 